\title{
Predictors of unanticipated admission within 30 days of outpatient sinonasal surgery*
}

\author{
Isabelle Gengler ${ }^{1,2}$, Laurent Carpentier ${ }^{3}$, Xavier Pasquesoone', \\ Dominique Chevalier', Geoffrey Mortuaire ${ }^{1,2}$
}

Otorhinolaryngology - Head and Neck Surgery Department, University Hospital, Lille, France

2 Lille Inflammation Research International Center - Inserm U995, Université de Lille, Lille, France

${ }^{3}$ Department of Anaesthesiology, University Hospital, Lille, France
Rhinology 55: 274-280, 2017

https:://doi.org/10.4193/Rhino16.251

*Received for publication:

August 29,2016

Accepted: October 2, 2016

Abstract
Objectives: To identify predictive factors of readmission after day-case rhinologic surgery.

Methods: A 2-year retrospective chart review of patients scheduled for ambulatory sinonasal surgery in a tertiary medical center was conducted. The operating room and the anesthetic files were screened to identify demographic data, types of procedure, comorbidities and post-operative complications.

Results: From January 2014 to January 2016, 924 outpatient sinonasal procedures were identified. The overall readmission rate within the 30-postoperative days was 5.1\% (2.9\% for overnight hospital stay, $2.2 \%$ for unplanned post procedure visit to the hospital via the emergency room, or directly to the surgical unit within 30 days of discharge). Age $\geq 50$ years, surgical duration $\geq$ $80 \mathrm{~min}$, endoscopic sinus surgery procedures and postoperative nasal packing were identified as negative predictive factors of readmission.

Conclusion: Careful scheduling of those higher-risk patients undergoing sinonasal surgery and appropriate postoperative observation should be implemented to improve healthcare quality in an outpatient setting.

Key words: ambulatory surgery, sinus surgery, septoplasty, readmission, day-case

\section{Introduction}

Ambulatory surgery accounts for a large and ever-increasing share of surgical procedures. Overall, more than $50 \%$ of elective surgeries are performed in an outpatient setting. Advances in non-invasive surgical techniques, innovations in anesthetic agents with decreased side effects and improvements in perioperative pain management and anti-emetic therapy have allowed caregivers to perform more complex surgical procedures and to operate on older, higher-risk patients in a day-case setting ${ }^{(1)}$. Day-case surgery offers shorter waiting time for surgery, lower risk of cross infection, less disruption of routine and a more rapid social and emotional rehabilitation (2).

Although sinus and nasal surgeries are increasingly performed in the ambulatory setting, rather limited data exists to assess outpatient surgical outcomes in rhinology ${ }^{(3)}$. Beyond sympto- matic recovery still being considered as the most important outcome for the patients ${ }^{(4)}$, limited unexpected hospital admission has been considered as a marker of performance and a standard of patient satisfaction ${ }^{(1,5)}$. The overall unplanned admission rate (encompassing overnight hospital stay and post procedure visit with admission) ranges from $2.65 \%$ to $8.8 \%$ and $13.1 \%$ in recent reported data ${ }^{(6-8)}$. Even though the correlation between unanticipated hospital admission and surgical quality is inconsistent, low admission rates have been adopted as a surrogate for healthcare quality without additional costs ${ }^{(9,10)}$. Academic guidelines for day-case surgery in rhinology have been issued to help standardization of procedures and patient selection ${ }^{(11,12)}$. Few studies from outpatient institutions are available to address risk factors of readmission in sinonasal surgery ${ }^{(6,7,13,14)}$. We set out on a 2-year retrospective chart review study of 
patients scheduled for ambulatory sinonasal surgery in a tertiary medical center to assess unplanned admission rates and to define predictive factors for unanticipated admission. Our objective is to better target those patients whose treatment would be suitable for outpatient sinonasal surgery.

\section{Materials and methods}

\section{Patients}

The study was conducted at the outpatient clinic at the Lille University Medical Center. We retrospectively included all patients undergoing day-case surgery for a sinonasal disease during the 24-month period from January 1, 2014 to January 1, 2016.

A procedure is defined as outpatient if the individual arrives and is discharged from the surgery facility on the same day the procedure was performed. This cohort study was approved by the CNIL Institutional Ethics Committee for medical data mining. Using the operating room database and the anesthetist files (which record the type of surgery and admission status), 924 ambulatory surgical procedures were identified for the study. All clinical data (e.g., patient age, gender, Body Mass Index (BMI), active smoking status, concomitant medical disease, chronic antithrombotic treatment) were acquired by systematic chart review. Comorbidity was scored according to the American Society of Anesthesiology (ASA) score. In our practice, patients with an ASA score of 1 (normal healthy patients) or an ASA score of 2 (patient with mild systematic disease) were eligible for an outpatient procedure. Patients with an ASA score $\geq 3$ were systematically referred to the inpatient service. To maintain patient confidentiality, cases were assigned a unique identifier number, and at no time were hospital-based identifiers used on data extraction forms or in the databases generated from chart review.

\section{Surgical procedures}

All patients were treated under general anesthesia in dedicated operating rooms for day-case surgery. All procedures were completed before 2:00 pm. Based on the International Classification of Diseases, Tenth Revision, four groupings of sinonasal procedures were assembled: endoscopic sinus surgery (ESS), rhinoplastic surgery, septoplasty and/or turbinate surgery and closed reduction of nasal bone fracture. For each patient, the length of procedure was reported. Nasal packing was used the discretion of the individual surgeon. Algosteril ${ }^{\mathrm{TM}}$ (Brothier Inc, Nanterre, France) or Merocel ${ }^{\mathrm{TM}}$ (Medtronic Inc, Dublin, Ireland) were used for ESS. Merocel ${ }^{\mathrm{TM}}$ was exclusively used during the other 3 procedures. Postoperatively, patients were monitored in the post-anesthesia care unit and then the ambulatory surgical unit. Patients were discharged from the ambulatory surgical unit when they met appropriate discharge criteria according to the post-anesthesia discharge scoring system. This score is based upon the recovery of vital signs without active bleeding and the control of pain, nausea and vomiting ${ }^{(14)}$. Distance traveled between home and the ambulatory surgical unit was reported.

\section{Outcome measurements}

The primary outcome of interest was unanticipated admission within 30 days of surgery. Unanticipated admission was defined as either a patient not discharged home on the day of surgery as planned, or a patient who was readmitted to the hospital (via the emergency room (ER), or directly to the surgical unit) within 30 days of discharge for a postoperative occurrence likely related to the principal surgical procedure. The reasons for admission and additional information were obtained from the case notes of re-admitted patients. They were classified as surgical (e.g., bleeding, pain, intracranial or orbital complications requiring observation) or anesthetic (e.g., nausea, vomiting, dizziness, somnolence, perioperative cardiac or pulmonary events). The admission of patients when their post-surgery escorts home did not show, were considered as social reasons for readmission, not medical.

\section{Statistical analysis}

Data were input into Microsoft Excel ${ }^{\mathrm{TM}}$ and statistical analysis was performed with SPSS ${ }^{\mathrm{TM}} \mathrm{v} 22.0$ statistical software (SPSS Inc., Chicago, IL, USA). Mean and standard deviation were reported for descriptive statistics. The Chi-squared test was used for comparison of frequency distribution. Independent categorical data were entered in a binary logistic regression model to evaluate their impact on unanticipated admission. Goodness of fit was assessed using the Hosmer-Lemeshow test. Size of effect was reported as adjusted odds ratios (ORs) and $95 \%$ confidence intervals (Cls). A two-tailed $p$-value $<0.05$ was considered statistically significant.

\section{Results}

General characteristics

During the study period, 924 sinonasal outpatient procedures under general anesthesia were performed (sex ratio $=514 \mathrm{M}$ / 410 F). The mean age was $37 \pm 16$ years. Active smoking habits were observed in 245 patients (26.5\%). All patients were classified as ASA I and II. A BMI $\geq 30$ kg.m-2 was reported in 116 patients (12.5\%). Comorbidities were associated in 347 patients (37.5\%). The most prevalent were hypertension (37.6\%), asthma (29.7\%), dyslipidemia (17.5\%), and diabetes (7.5\%). Antithrombotic treatments were prescribed in 46 patients (5\%).

\section{Surgical procedures}

There were 488 endoscopic sinus surgeries (53\%), 256 septoplasty and/or turbinate surgeries (28\%), 94 closed reduction of nasal bone fractures (10\%), and 86 rhinoplastic surgeries (9\%) (Table 1). ESS consisted of 260 middle meatotomies (53\%), 139 anterior ethmoidectomies (28.5\%), 47 complete unilateral sphenoethmoidectomies (10\%), 32 complete bilateral sphenoet- 
Table 1. Preoperative versus postoperative scores.

\begin{tabular}{|c|c|c|c|c|c|}
\hline \multicolumn{2}{|c|}{ Surgical procedures } & ESS & $\begin{array}{l}\text { Septoplasty and } \\
\text { /or turbinoplasty }\end{array}$ & $\begin{array}{l}\text { Closed reduction } \\
\text { of nasal bone } \\
\text { fracture }\end{array}$ & $\begin{array}{l}\text { Rhinoplastic } \\
\text { surgery }\end{array}$ \\
\hline Population & & 488 & 256 & 94 & 86 \\
\hline Duration of surgery (min) & & $51( \pm 32)$ & $69( \pm 33)$ & $21( \pm 10)$ & $86( \pm 33)$ \\
\hline Postoperative nasal packing (\%) & & 20 & 93 & 83 & 99 \\
\hline Schedule of nasal packing removing & $\begin{array}{l}3 \mathrm{~h} \\
24 \mathrm{~h} \\
48 \mathrm{~h} \\
>48 \mathrm{~h}\end{array}$ & $\begin{array}{c}62 \\
7 \\
22 \\
7\end{array}$ & $\begin{array}{c}4 \\
13 \\
173 \\
49\end{array}$ & $\begin{array}{c}1 \\
- \\
47 \\
30\end{array}$ & $\begin{array}{c}2 \\
3 \\
50 \\
30\end{array}$ \\
\hline Nasal bleeding occurence & $\begin{array}{l}\text { Before day-case discharge } \\
\text { After day-case discharge }\end{array}$ & $\begin{array}{l}6 \\
4\end{array}$ & $\begin{array}{l}1 \\
4\end{array}$ & - & - \\
\hline
\end{tabular}

ESS $=$ Endoscopic Nasal Surgery. Duration of surgery is expressed as mean \pm SD.

hmoidectomies (6.5\%), and 10 Draf III frontal sinotomies (2\%). Rhinoplastic surgeries consisted of 50 closed procedures (58\%) and 36 open procedures (42\%). The mean duration of surgery was $56 \otimes 35 \mathrm{~min}$ with a range of $21 \pm 10 \mathrm{~min}$ for closed reduction of nasal bone fractures to $86 \pm 33 \mathrm{~min}$ for rhinoplastic surgery. Nasal packing was used in $99 \%$ of rhinoplastic surgery, $93 \%$ of septoplasty \pm turbinate surgery, and $83 \%$ of closed reduction of nasal bone fractures. For the three aforementioned procedures, nasal packing was removed two days later. Nasal packing was used postoperatively in $20 \%$ of endoscopic sinus surgeries, typically for a duration of 3 hours (Table 1). After discharge, the mean distance of travel from the outpatient unit to the patient home was $32 \pm 30 \mathrm{~km}$ (from 3 to $163 \mathrm{~km}$ ).

\section{Outcome measurements}

During the 24-month study, 50 unanticipated admissions were reported (5.1\%). Using univariate analysis (Table 2), patients with unanticipated admission were older (48 yrs vs 39 ), and their surgical procedures were longer ( $75 \mathrm{~min}$ vs 43 ). Among those 50 unanticipated admissions, 27 were not discharged the same day (2.9\%) and 23 were discharged the same day but were subsequently readmitted to the emergency room or the rhinologic surgical unit during the 30-day postoperative period (2.5\%) (Table 3). Overall, the reasons for unanticipated admission were surgical in 33 cases (bleeding (15), pain (7), and intolerance to the nasal packing or dressing (6) were chiefly reported), and anesthetic in 11 cases (postoperative nausea and vomiting (3),

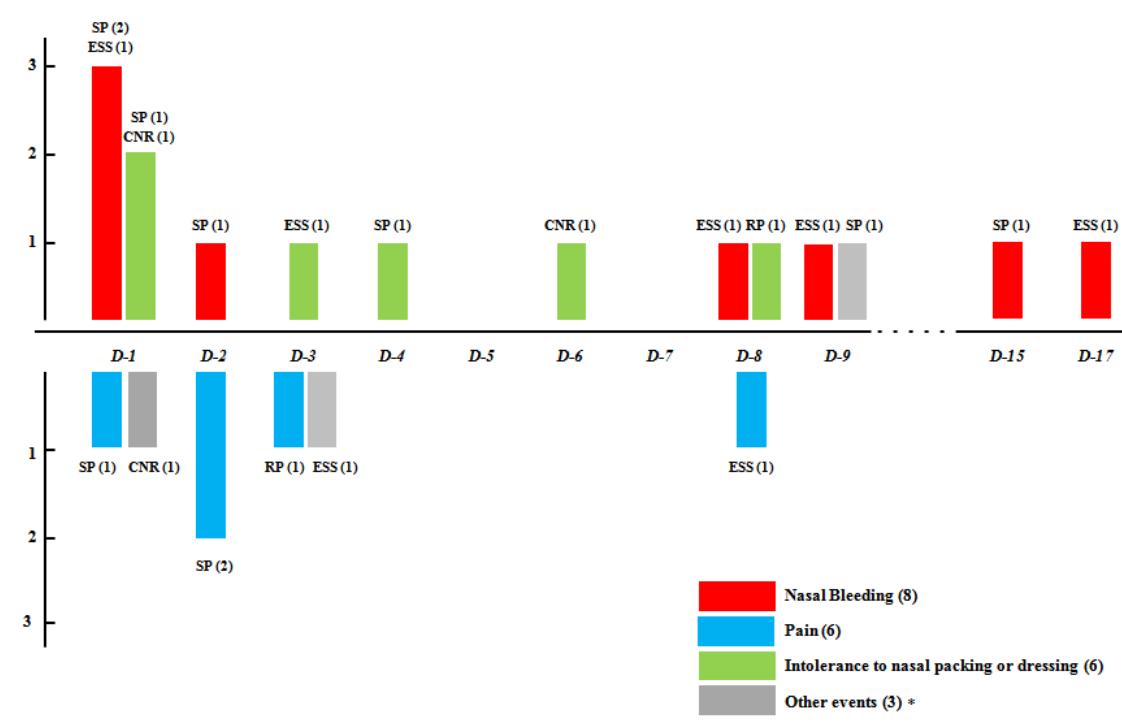

Figure 1. Chronology of post procedure visits to the emergency room within 30 days. Readmissions are depicted in function of time. The type of event leading to visit to the emergency room is represented by a colored bar. For each bar, the type and the number of surgical procedures are specified. ESS = Endoscopic Sinus Surgery, SP = Septoplasty, CNR = Closed Reduction of Nasal bone fracture, RP = Rhinoplastic surgery. $\left(^{*}\right)$ Other events refer to fever (day 1), intolerance to codeine (day 3), and septum hematoma (day 9). 
Table 2. Characteristics of patients studied.

\begin{tabular}{|c|c|c|c|c|c|c|c|}
\hline & \multicolumn{3}{|c|}{ Same-day discharge $(n=874)$} & \multicolumn{3}{|c|}{ Unanticipated admission $(n=50)$} & \multirow[t]{2}{*}{$\mathbf{p}$} \\
\hline \multicolumn{7}{|l|}{ Preoperative } & \\
\hline $\operatorname{Sex}(n)$ & M: 484 & & $\mathrm{~F}: 390$ & M: 30 & & $\mathrm{~F}: 20$ & ns \\
\hline Age (yr) & $39( \pm 16)$ & & & $48( \pm 17)$ & & & 0.02 \\
\hline Body Mass Index (kg.m-2) (\%) & $\begin{array}{l}\leq 30 \\
87.9\end{array}$ & & $\begin{array}{l}>30 \\
12.1\end{array}$ & $\begin{array}{c}\leq 30 \\
80\end{array}$ & & $\begin{array}{c}>30 \\
20\end{array}$ & ns \\
\hline Active smoking (\%) & 26.7 & & & 22 & & & ns \\
\hline Concomitant medical diseases (\%) & $\begin{array}{c}0 \\
58.6\end{array}$ & $\begin{array}{c}\leq 2 \\
36.8\end{array}$ & $\begin{array}{l}>2 \\
4.6\end{array}$ & $\begin{array}{c}0 \\
50\end{array}$ & $\begin{array}{l}\leq 2 \\
44\end{array}$ & $\begin{array}{c}>2 \\
6\end{array}$ & ns \\
\hline Respiratory disorder (\%) & 14.7 & & & 28.2 & & & ns \\
\hline Antithrombotic drugs (\%) & 4.9 & & & 6 & & & ns \\
\hline \multicolumn{8}{|l|}{ Intraoperative } \\
\hline Endoscopic sinus surgery (\%) & 52.3 & & & 62 & & & ns \\
\hline Surgery duration (min) & $55( \pm 34)$ & & & $75( \pm 43)$ & & & 0.002 \\
\hline \multicolumn{8}{|l|}{ Postoperative } \\
\hline Nasal packing (\%) & 46.1 & & & 62 & & & ns \\
\hline Distance traveled (km) & $37( \pm 25)$ & & & $27( \pm 54)$ & & & ns \\
\hline
\end{tabular}

According to the number of concomitant medical diseases, the patients were divided in 3 groups: without concomitant medical disease, with 1 or 2 concomitant medical diseases, with more than 2 associated diseases. Respiratory disorder refers to chronic obstructive pulmonary disease, asthma and obstructive sleep apnea. Age, surgery duration and distance traveled are expressed as mean \pm SD. ns = non-significant $p$ value

Table 3. Reasons for unanticipated admission (50 patients).

Overnight stay with admission to hospital as inpatient: $\mathrm{n}=\mathbf{2 7}(2.9 \%)$

\begin{tabular}{|c|c|c|c|}
\hline Surgical: $n=12(44 \%)$ & Anesthetic: $n=10(37 \%)$ & Social: $n=4$ (15\%) & Other: $\mathrm{n}=1$ (3\%) \\
\hline $\begin{array}{l}\text { Bleeding }(7) \\
\text { Skull base repair (4) } \\
\text { Pain (1) }\end{array}$ & $\begin{array}{l}\text { PONV( 3) } \\
\text { Dizziness/somnolence (3) } \\
\text { Thoracic pain (2) } \\
\text { Bronchospasm (1) } \\
\text { Post intubation lingual oedema (1) }\end{array}$ & $\begin{array}{l}\text { No escort (3) } \\
\text { Patient request (1) }\end{array}$ & Late operating time (1) \\
\hline
\end{tabular}

\section{Bleeding (8)}

Intolerance to nasal packing or

dressing (6)

Pain (6)

Septum hematoma (1)

Fever (1)

Intolerance to codeine (1)

PONV = postoperative nausea and vomiting. Data for each complication presented as counts. Among the 27 patients who were not discharged the same day, 24 were treated with ESS. The other 3 were treated with septoplasty and were admitted for somnolence ( 1 patient), bronchospasm (1 patient). One patient had no escort.

dizziness/somnolence (3)). Nasal bleeding occurred within the first 3 hours postoperatively in 7 patients leading to subsequent hospitalization. Nasal bleeding occurred 24 hours after surgery in 3 patients, two days later in 1 patient, and after the eighth day in 4 patients (Table 1 and Figure 1). No patient escort was available in 3 of the cases. One patient asked to stay overnight for personal reasons. In one case, the patient left the postanesthesia care unit too late for adequate same-day discharge. A subsequent surgical procedure was required for only one patient with a postoperative septum hematoma.

When comparing outpatient cases with unanticipated admission cases using a binary logistic regression model (with unanticipated admission as the dependent variable), we observed that patients who were older than $50(\mathrm{OR}=2.0)$, patients whose surgery lasted longer than $80 \mathrm{~min}(\mathrm{OR}=2.53)$, patients who had undergone endoscopic sinus surgery $(\mathrm{OR}=5.51)$ and patients who required the use of nasal packing $(\mathrm{OR}=5.36)$ demonstrated statistically a higher risk of unanticipated admission (Table 4). 
Table 4. Summary of results from binary logistic regression for unanticipated admission.

\begin{tabular}{|c|c|c|c|c|}
\hline Independent Variables & Level & Odds Ratio & $95 \% \mathrm{Cl}$ & $\mathbf{p}$ \\
\hline \multicolumn{5}{|l|}{ Preoperative } \\
\hline $\operatorname{Sex}(n)$ & $\begin{array}{l}\text { Male } \\
\text { Female }\end{array}$ & $\begin{array}{c}\text { Reference } \\
0.81\end{array}$ & $0.43-1.49$ & 0.49 \\
\hline Age (yr) & $\begin{array}{l}<50 \\
\geq 50\end{array}$ & $\begin{array}{c}\text { Reference } \\
2.00\end{array}$ & $1.10-4.01$ & 0.05 \\
\hline Body Mass Index (kg.m-2) (\%) & $\begin{array}{l}\leq 30 \\
87.9\end{array}$ & $\begin{array}{c}\text { Reference } \\
1.94\end{array}$ & $0.81-4.6$ & 0.13 \\
\hline Preoperative disease & $\begin{array}{l}\text { No } \\
\text { Yes }\end{array}$ & $\begin{array}{c}\text { Reference } \\
1.09\end{array}$ & $0.57-2.1$ & 0.78 \\
\hline Antithrombotic drug & $\begin{array}{l}\text { No } \\
\text { Yes }\end{array}$ & $\begin{array}{c}\text { Reference } \\
0.72\end{array}$ & $0.19-2.71$ & 0.63 \\
\hline \multicolumn{5}{|l|}{ Intraoperative } \\
\hline Endoscopic sinus surgery (\%) & $\begin{array}{l}\text { No } \\
\text { Yes }\end{array}$ & $\begin{array}{c}\text { Reference } \\
5.51\end{array}$ & $2.19-13.8$ & $<0.001$ \\
\hline Surgery duration (min) & $\begin{array}{l}<80 \\
\geq 80\end{array}$ & $\begin{array}{c}\text { Reference } \\
2.53\end{array}$ & $1.36-4.69$ & 0.003 \\
\hline \multicolumn{5}{|l|}{ Postoperative } \\
\hline Nasal packing (\%) & $\begin{array}{l}\text { No } \\
\text { Yes }\end{array}$ & $\begin{array}{c}\text { Reference } \\
5.36\end{array}$ & $2.19-13.1$ & $<0.001$ \\
\hline Distance traveled $(\mathrm{km})$ & $\begin{array}{l}<50 \\
\geq 50\end{array}$ & $\begin{array}{c}\text { Reference } \\
0.53\end{array}$ & $0.24-1.19$ & 0.12 \\
\hline
\end{tabular}

$\mathrm{Cl}=$ confidence interval; Hosmer Lemeshow Goodness-of-fit Test: $\mathrm{p}$ value $=0.82$

\section{Discussion}

The quality and efficacy of day-case surgical care is predicated upon careful selection of patients, minimization of complication rates, and avoidance of inpatient service disruption as a result of unexpected admissions ${ }^{(2)}$.

Several studies have been published to assess unplanned admission in the ambulatory setting after various types of surgery $(1,10,16-19)$. The highest rates of unplanned admission occurred with ear, nose and throat (ENT) surgery, followed by urology ${ }^{(17,18)}$. In a dedicated ENT day-care unit, Ganesan et al. showed that most admissions were due to hemorrhage following sinonasal surgery ${ }^{(2)}$. Recent data from outpatient sinonasal surgeries are available, but populations were either limited to specific procedures (endoscopic sinus surgery, septoplasty) ${ }^{(7,13)}$ or spanned a long period of time with modifications of surgical procedures ${ }^{(14)}$. A large multicenter cohort was built to achieve strong results, but local anesthetic alone was used for most of the outpatient procedures ${ }^{(20)}$. As reported by Bhattacharyya ${ }^{(6)}$, the individual databases in multicenter studies were dependent on institutional submission of data; therefore they did not encompass $100 \%$ of all ambulatory sinonasal surgeries. Herein, we chose to focus our analysis on the most recent standardized data available to obtain the most homogenous data regarding surgical practices and patient medical management in one institution. As recommended by Rudmik et al., surgery was implemented in each case after conducting optimal medical treatment and after informed consent was obtained ${ }^{(21)}$. All procedures were performed under general anesthesia as a standard for ambulatory surgery. Accuracy of information was achieved by cross-referencing surgical, anesthetic, and institutional databases. In an effort to avoid under-reporting of follow-up care, direct phone calls from nurses to patients within 24 hours of discharge were implemented as standard practice.

The present study found that our rate of overnight admission after ambulatory surgery (2.9\%) is consistent with previous studies $(1,6,22)$. As previously observed ${ }^{(6)}$, there is very little data regarding revisit and readmission rates beyond the immediate 24-hour window. We extended our analysis to the 30th postoperative day to provide a broader metric for the unanticipated used of healthcare resources. Scheduled postoperative follow-up visits and physician's office visits for other reasons were not included in this analysis. In our study, unanticipated admission rate to the ER and surgical rhinologic unit beyond the first day was $2.5 \%$. A $5 \%$ rate was described for unplanned admissions up to 14 days after the sinonasal ambulatory procedure in a US multi-state review ${ }^{(6)}$. De Oliveira et al. observed a $2.5 \%$ rate of readmission within 30 days of ambulatory surgery ${ }^{(10)}$.

As reported $(6,14,17)$, the largest reason for admission was surgical, with bleeding in $30 \%$ of cases. From a preventive care standpoint, better pre- and post-operative information on what does and does not constitute serious bleeding may significantly reduce the number of ER visits ${ }^{(18)}$. In our study, all of the patients with bleeding were discharged from the ER without admission 
to the inpatient hospital setting, as bleeding was considered minor. Pain was the second most frequent reason for readmission in $14 \%$ of cases. This rate is low considering the standard anesthetic requirements of day-case surgery. Nevertheless, pain management efforts should not only focus on effective control of pain in the hospital, but also on the anticipation of pain-related issues upon discharge ${ }^{(14,23)}$. Anesthetic reasons (nausea, vomiting and somnolence) accounted for $37 \%$ of overnight stays, confirming the findings of previous studies ${ }^{(17)}$. It is reasonable to expect that admission for these reasons could be prevented by improving the quality of recovery.

We believe that better preoperative screening of patients can reduce the incidence of both overnight stay and readmission after outpatient procedure. More effective preoperative screening would also better prepare both the patient and the hospital for the likelihood of inpatient stay following surgery ${ }^{(13)}$. With a binary logistic regression model, we showed that age, surgical length, sinus surgery procedures and nasal packing were predictive factors for unanticipated admission. In a multivariate analysis of 53,667 ambulatory surgical cases, De Oliveira et al. showed that older adults were at greater risk of unanticipated hospital admission ${ }^{(10)}$. Cognitive impairment after anesthesia with greater comorbidities reduces their capacity to return to daily function after ambulatory surgery. Length of surgery is also recognized as a risk factor of unanticipated admission ${ }^{(1,24)}$. These findings likely implicate intraoperative complications like excessive bleeding or skull base injuries and delayed recovery after anesthesia ${ }^{(17)}$. Revision cases with extensive surgery could also be involved in prolonged surgical procedure ${ }^{(7,25)}$. In our study, $15 \%$ of overnight stay was due to unplanned extensive surgery with skull base repair for meningocele (1 pt), frontal mucocela (1 pt), T1 ethmoidal adenocarcinoma (1 pt), and extensive sphenoid sinusitis (1 pt). As a result, sinus surgery was associated with a higher risk of unanticipated admission in comparison with other nasal procedures. In a retrospective chart review of 194 patients with sinus surgery, Lin et al. reported an unexpected admission rate of $4.7 \%$, which is higher than rates obtained for overall nasal procedures ${ }^{(3,13)}$.

The impact of nasal packing is controversial. It was previously considered as preventing earlier discharge given that removal time was often planned for the following day ${ }^{(26)}$. In our study, the presence of nasal packing was a predictive factor of unanticipated admission. In a study comparing patients with different nasal packs time of removal, Tierney et al. showed that the patients' acceptance for day-case sinonasal surgery was directly correlated to a shorter time of removal (27). Selective use of nasal packs seems to reduce patient morbidity and facilitate day-case surgery. That being said, the risk of postoperative bleeding or hematoma needs to be balanced with patient expectation. We did not find that medical comorbidities were linked to unanticipated admission. Our results are consistent with the En- glish audit of day-case septoplasty ${ }^{(7)}$. One explanation of these findings is the preoperative selection of patients eligible for day-case surgery. In our institution, ASA class I-II was a restricted standard for sinonasal ambulatory surgery. Only patients with mild OSA treated with PAP, controlled asthma and other mild chronic respiratory diseases were selected. The same conclusion can be drawn for cardiovascular and metabolic diseases. The impact of obesity is more controversial. Hofer et al. showed that obesity was not a significant independent risk factor of unanticipated admission in a study comparing 235 obese patients matched with control patients for ambulatory surgery ${ }^{(19)}$. Others case-control studies reported obesity as a potential risk factor $(1,17)$. This discrepancy can be explained by biased population selection with some studies including patients with ASA class III-IV patients. In our study, active smoking was evenly distributed in both groups of patient with or without unanticipated admission and was not considered as risk factor of overnight stay in previous univariate and multivariate studies ${ }^{(1,13,17)}$. The role of antithrombotic drugs was not evaluated in recent studies. We did not show negative impact in selected patients.

\section{Conclusion}

Advances in sinonasal surgical procedures and anesthetic techniques have increased patient eligibility for ambulatory rhinologic surgery. With more than $50 \%$ of elective sinonasal surgeries taking place in an outpatient setting, and an overnight stay rate of $2.9 \%$, our data are consistent with international standards. The present study involving a relatively large cohort of day-case surgery patients suggests that predictive factors of unanticipated admission can be targeted to match patient selection and pathology management. Careful scheduling of higher-risk patients, appropriate postoperative observation and adequate patient information are key predictors of high-standard recovery and patient satisfaction.

\section{Acknowledgements}

The authors would like to thank Dr Didier Theis, Dr Guillaume Clément and Mr Xavier Lenne from the medical information department for their expert technical assistance in data collection. The authors also wish to thank Mr Gerald Vuolo for language reviewing.

\section{Authorship contribution}

IG: acquisition of data, analysis of data, interpretation of data; LC: acquisition of data; XP: acquisition of data; DC: conception and design; GM: conception and design, analysis and interpretation of data, drafting of the article, final approval of the version to be submitted.

\section{Conflict of interest}

None to declare. 


\section{References}

1. Whippey A, Kostandoff G, Paul J, Ma J, Thabane L, Ma HK. Predictors of unanticipated admission following ambulatory surgery: a retrospective case-control study. Can J Anaesth J Can Anesth 2013; 60:675-683.

2. Ganesan S, Prior AJ, Rubin JS. Unexpected overnight admissions following day-case surgery: an analysis of a dedicated ENT day care unit. Ann R Coll Surg Engl 2000; 82:327-330

3. Bhattacharyya N. Ambulatory sinus and nasal surgery in the United States: demographics and perioperative outcomes. Laryngoscope 2010; 120:635-638.

4. Hopkins C, Philpott C, Crowe S, et al. Identifying the most important outcomes for systematic reviews of interventions for rhinosinusitis in adults: working with Patients, Public and Practitioners. Rhinology 2016;54:20-26.

5. Joynt KE, Jha AK. A path forward on Medicare readmissions. N Engl J Med 2013 368:1175-1177.

6. Bhattacharyya N. Unplanned revisits and readmissions after ambulatory sinonasal surgery. Laryngoscope 2014; 124:1983-1987.

7. Georgalas C, Obholzer R, Martinez-Devesa P, Sandhu G. Day-case septoplasty and unexpected re-admissions at a dedicated daycase unit: a 4-year audit. Ann R Coll Surg Engl 2006; 88:202-206.

8. Eisenberg G, Pérez $C$, Hernando $M$ et al. [Nasosinusal endoscopic surgery as major out-patient surgery]. Acta Otorrinolaringológica Esp 2008; 59:57-61.

9. Morris MS, Deierhoi RJ, Richman JS, Altom LK, Hawn MT. The relationship between timing of surgical complications and hospital readmission. JAMA Surg 2014; 149:348-354.

10. De Oliveira GS, Holl JL, Lindquist LA, Hackett NJ, Kim JYS, McCarthy RJ. Older Adults and Unanticipated Hospital Admission within 30 Days of Ambulatory Surgery: An Analysis of 53,667 Ambulatory Surgical Procedures. J Am Geriatr Soc 2015; 63:1679-1685.

11. de Gabory L, Serrano E, Lecanu J-B, et al. French Otorhinolaryngology Society guide- lines for day-case nasal surgery. Eur Ann Otorhinolaryngol Head Neck Dis 2015; 132:35-40.

12. Audet N, Leclerc JE, Iordanescu V Consensus on ambulatory care among Quebec otolaryngologists. J Otolaryngol 1999; 28:43-48.

13. Lin D, Dalgorf D, Witterick IJ. Predictors of unexpected hospital admissions after outpatient endoscopic sinus surgery: retrospective review. J Otolaryngol - Head Neck Surg J Oto-Rhino-Laryngol Chir CervicoFaciale 2008; 37:309-311.

14. Wong A, Kacker A. Incidence of unplanned admissions after sinonasal surgery: a 6-year review. Int Forum Allergy Rhinol 2014; 4:143-146.

15. Chung F. Discharge criteria--a new trend. Can J Anaesth J Can Anesth 1995; 42:1056-1058

16. Bryson GL, Gomez CP, Jee RM, Blackburn J, Taljaard M, Forster AJ. Unplanned admission after day surgery: a historical cohort study in patients with obstructive sleep apnea. Can J Anaesth J Can Anesth 2012; 59:842-851.

17. Fortier J, Chung F, Su J. Unanticipated admission after ambulatory surgery--a prospective study. Can J Anaesth J Can Anesth 1998; 45:612-619.

18. Twersky R, Fishman D, Homel P. What happens after discharge? Return hospital visits after ambulatory surgery. Anesth Analg 1997; 84:319-324.

19. Hofer RE, Kai T, Decker PA, Warner DO. Obesity as a risk factor for unanticipated admissions after ambulatory surgery. Mayo Clin Proc 2008; 83:908-916.

20. Hopkins C, Browne JP, Slack R, et al. The national comparative audit of surgery for nasal polyposis and chronic rhinosinusitis. Clin Otolaryngol Off J ENT-UK Off J Neth Soc Oto-Rhino-Laryngol Cervico-Facial Surg 2006; 31:390-398

21. Rudmik L, Soler ZM, Hopkins C, et al. Defining appropriateness criteria for endoscopic sinus surgery during management of uncomplicated adult chronic rhinosinusitis: a RAND/UCLA appropriateness study.
Rhinology 2016;54(2):117-128.

22. Stessel B, Fiddelers AA, Joosten EA, Hoofwijk DMN, Gramke H-F, Buhre WFFA. Prevalence and Predictors of Quality of Recovery at Home After Day Surgery. Medicine (Baltimore) 2015; 94:e1553.

23. Coley KC, Williams BA, DaPos SV, Chen C, Smith RB. Retrospective evaluation of unanticipated admissions and readmissions after same day surgery and associated costs. J Clin Anesth 2002; 14:349-353.

24. Tewfik MA, Frenkiel S, Gasparrini R, et al. Factors affecting unanticipated hospital admission following otolaryngologic day surgery. J Otolaryngol 2006; 35:235-241.

25. Hopkins C, Browne J, Slack R, Brown P. Variation in day-case nasal surgery - why cannot we improve our day-case rates? Clin Otolaryngol Off J ENT-UK Off J Neth Soc Oto-Rhino-Laryngol Cervico-Facial Surg 2007; 32:12-18.

26. Eliashar R, Gross M, Wohlgelernter J, Sichel $J$-Y. Packing in endoscopic sinus surgery: is it really required? Otolaryngol--Head Neck Surg Off J Am Acad Otolaryngol-Head Neck Surg 2006; 134:276-279.

27. Tierney PA, Samuel D, Patel KS, Thomas DM Audit of patient acceptance of nasal surgery as a day case procedure. Br J Clin Pract 1996; 50:357-359.

Geoffrey Mortuaire, MD, PhD Service d'ORL de chirurgie cervico-faciale, Hôpital Huriez CHRU Lille, 59000

France

Tel: (+33) 320445675

Fax: (+33) 320446220

E-mail: geoffrey.mortuaire@chru-lille.fr 